\title{
DIVERSIDAD HERPETOFAUNÍSTICA DEL PARQUE NACIONAL LAGUNAS DE CHACAHUA Y LA TUZA DE MONROY, OAXACA, MÉXICO
}

\author{
Jesús GARCÍA-GRAJALES ${ }^{1 *}$, Alejandra BUENROSTRO-SILVA² \& Vicente MATA-SILVA ${ }^{3}$
}

\author{
$1 *$ Instituto de Recursos, Universidad del Mar. Campus Puerto Escondido, Km 2.5, carretera Puerto Escondido- \\ Sola de Vega, San Pedro Mixtepec 71980, Oaxaca, México. <archosaurio@yahoo.com.mx> \\ ${ }^{2}$ Instituto de Industrias, Universidad del Mar. Campus Puerto Escondido, Km 2.5, carretera Puerto Escondido-Sola \\ de Vega, San Pedro Mixtepec 71980, Oaxaca, México.<sba_1575@yahoo.com.mx> \\ ${ }^{3}$ The University of Texas at El Paso, Department of Biological Sciences. El Paso, Texas 79968, USA. \\ <vimasil@yahoo.com>
}

Recibido: 15/06/2015; aceptado: 19/01/2016

García-Grajales, J., Buenrostro-Silva, A. \& Mata-Silva, V. 2016. Diversidad herpetofaunística del Parque Nacional Lagunas de Chacahua y La Tuza de Monroy, Oaxaca, México. Acta Zoológica Mexicana (n. s.), 32(1): 90-100.

RESUMEN. Se realizó un listado de especies de anfibios y reptiles que habitan en el interior del Parque Nacional Lagunas de Chacahua (PNLCh) y La Tuza de Monroy, se analizó cuantitativamente su riqueza específica y abundancia relativa. El trabajo se llevó a cabo de manera mensual y alternada para cuatro localidades entre los meses de febrero del 2009 y febrero de 2010, abarcando la temporada de sequía y lluvias. Se construyeron curvas de acumulación de especies basados en el modelo de Chao2 y el comportamiento de las especies únicas y dobles. En total se registraron 52 especies distribuidas en 47 géneros y 26 familias. La mayor diversidad de los anfibios $\left(H^{\prime}=0.98\right)$ se obtuvo en la época de lluvias mientras que en el caso de los reptiles $\left(H^{\prime}=\right.$ 1.14) fue en la época de sequía. La prueba de Hutcheson reveló que la diversidad de los anfibios entre épocas fue estadísticamente diferente $(t=1.22$, g. $1 .=406, P>0.05)$ mientras que en el caso de los reptiles no hubo diferencias significativas entre épocas $(t=3.07$, g. $1 .=489$, $P<0.05)$. Las curvas de intervalo-abundancia muestran un marcada dominancia de las especies comunes en sitios perturbados y de especies invasoras (Rhinella marina y Hemidactylus frenatus). Este trabajo representa una aportación al conocimiento de la herpetofauna de la planicie costera de Oaxaca y del PNLCh y el inicio de una base para estudios futuros sobre historia natural y ecología de este importante grupo de organismos en la costa de Oaxaca.

Palabras clave: Riqueza, dominancia, diversidad, anfibios, reptiles, Chacahua.

\section{INTRODUCCIÓN}

Oaxaca es el estado con mayor riqueza biológica del país en diferentes grupos taxonómicos de flora y fauna, debido a la amalgama de su accidentada topografía, las variaciones climáticas y la composición de su vegetación (Navarro et al. 2004, Torres-Colín 2004, Trejo 2004, Flores-Villela et al. 2005), ocupa el quinto lugar en extensión del país
García-Grajales, J., Buenrostro-Silva, A. \& Mata-Silva, V. 2016. Herpetofaunistic diversity of Parque Nacional Lagunas de Chacahua and La Tuza de Monroy, Oaxaca, Mexico. Acta Zoológica Mexicana (n. s.), 32(1): 90-100.

ABSTRACT. A list of amphibians and reptiles in the Parque Nacional Lagunas de Chacahua (PNLCh) and La Tuza de Monroy was accomplished; diversity and relative abundance were analyzed quantitatively. The work was carried out monthly and alternated for four locations between February 2009 and February 2010 comprising both drought and wet seasons. Species accumulation curves were constructed based on the Chao 2 model and the behavior of single and double species. Fifty two species were registered, distributed in 47 genera and 26 families. The greatest diversity of amphibians $\left(H^{\prime}=0.98\right)$ was obtained in the rainy season; while in the case of reptiles $\left(H^{\prime}=1.14\right)$ was during the drought season. The Hutcheson test revealed that the diversity of amphibians among seasons was statistically different $(t=1.22$, g. 1 . $=406, P<0.05)$ while there were no significant differences between seasons for reptiles $(t=3.07$, g. $1 .=489, P>0.05)$. Rank-abundance curves show a marked dominance by species common in disturbed sites and by invasive species (Rhinella marina and Hemidactylus frenatus). This work represents a contribution to the knowledge of the Oaxacan herpetofauna from the coastal plains and the PNLCh, and groundwork for future studies on natural history and ecology of this important group of organisms.

Key words: Richness, dominance, diversity, amphibians, reptiles, Chacahua.

y es una de las regiones con mayor riqueza específica de anfibios y reptiles del mundo (Mata-Silva et al. 2015). La herpetofauna en este estado representa aproximadamente el 36.4\% del total para México con un elevado porcentaje de endemismos (Casas-Andreu 1996, Mata-Silva et al. 2015), convirtiéndose en un grupo de gran relevancia, debido a que han encontrado las condiciones favorables para su desarrollo, además de jugar un papel importante 
en la cadena trófica y cumplir funciones y ciclos indispensables en el ecosistema (Mena-Correa 2008).

El conocimiento de la riqueza de especies de anfibios y reptiles de Oaxaca surgió desde 1824 con las colectas de Ferdinand Deppe, generándose desde entonces significativas aportaciones científicas en este campo de estudio (Casas-Andreu et al.1996, García-Grajales 2008); sin embargo, aun cuando el estudio de los anfibios y reptiles de Oaxaca se ha visto enriquecido significativamente en los últimos años, sigue existiendo una carencia de información para ciertas zonas en cuanto a la distribución y diversidad de especies, lo cual se ve reflejado en la falta de inventarios actualizados de estos organismos (CasasAndreu et al. 2004).

El Parque Nacional Lagunas de Chacahua (PNLCh), decretado en 1937, abarca una extensión de 13,264 hectáreas, de las cuales 10,331 corresponden a tierra firme y 2,902 a lagunas costeras (Pérez-Delgado 2002); constituye un área de gran importancia ecológica, económica, social, histórica y cultural que se caracteriza por una variedad de condiciones ambientales que favorecen la presencia de diversos hábitats (Hernández-Santos 2009); sin embargo, el conocimiento sobre los anfibios y reptiles que se distribuyen en el PNLCh sigue siendo escaso. Por otro lado, se ha demostrado que una Área Natural Protegida (ANP), por muy extensa que sea, no es posible que reúna toda la riqueza del paisaje, debido a que esta diversidad está espacialmente dispersa a lo largo de una región (Halffter 2005, 2007). En este sentido, existe la posibilidad de que la región de La Tuza de Monroy, área adyacente al PNL$\mathrm{Ch}$, sea un sitio prioritario para la conservación de varios grupos de vertebrados incluyendo los anfibios y reptiles de la región; por lo que generar listados de especies que proporcionen información sólida y confiable permitirá conocer la diversidad de anfibios y reptiles del PNLCh y áreas adyacentes.

El presente estudio aporta una lista de las especies de anfibios y reptiles presentes en el PNLCh, analizando cuantitativamente la riqueza y composición taxonómica y estimando la abundancia relativa de las especies que habitan en el área de estudio con el fin de sentar las bases para estudios posteriores sobre historia natural de estos vertebrados en esta región.

\section{MATERIALES Y MÉTODOS}

Área de estudio. El estudio se llevó a cabo dentro del polígono del PNLCh y en La Tuza de Monroy, Oaxaca.
E1 PNLCh se ubica al suroeste del estado de Oaxaca en la región costera del municipio de Villa de Tututepec de Melchor Ocampo, entre las coordenadas $16^{\circ} 04^{\prime} 10^{\prime \prime}$,

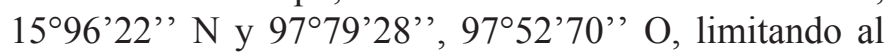
este con Zapotalito y Cerro Hermoso, al sur con el Océano Pacífico, al norte con San José del Progreso y al oeste con el Río Verde (Hernández-Santos 2009). La región de la Tuza de Monroy, se ubica de igual manera al suroeste del estado de Oaxaca en el municipio de Santiago Jamiltepec, entre las coordenadas $16^{\circ} 08^{\prime} 52^{\prime \prime}, 16^{\circ} 08^{\prime} 26^{\prime}$ ' $\mathrm{N} \mathrm{y}$ $97^{\circ} 58^{\prime} 02^{\prime}$ ', 9744'18' O, limitando al sureste con el Río Verde, al sur con el Océano Pacífico, al norte con la Sierra Madre del Sur y al oeste por el Río La Arena (Lira \& Naranjo 2005) (Figura 1).

La vegetación dominante de esta región corresponde a selva baja caducifolia, selva mediana subcaducifolia y subperennifolia (Rzedowski 1978, Torres-Colín 2004). El clima de la región de acuerdo a la clasificación de Köppen modificada por García (1988), es A Wo (w) i, g, que corresponde al grupo climático cálido con una temperatura media anual mayor a $28^{\circ} \mathrm{C}$ y la del mes más frío superior a $18{ }^{\circ} \mathrm{C}$ con lluvias concentradas, generalmente de julio a octubre. La precipitación anual es de aproximadamente 1000 mm (García 1988).

Trabajo de campo. Se llevó a cabo de manera mensual y alterna para cada sitio durante el periodo comprendido entre febrero de 2009 y febrero de 2010, exceptuando los meses de junio y julio de 2009, con una duración de cuatro días por muestreo para cada comunidad. Los muestreos siguieron un orden en cuanto a tiempo y esfuerzo horas/hombre, aplicado para cada uno de los cuatro sitios (La Tuza, El Azufre, El Corral y Cerro Hermoso). Los sitios se eligieron con base en su estado de conservación, la presencia de los tres tipos de vegetación natural de mayor dominancia y la facilidad para el acceso, con el consentimiento de los pobladores para el trabajo de campo, el cual incluyó la temporada de sequía y lluvias.

En cada muestreo se establecieron 20 líneas de desvío con una longitud de $10 \mathrm{~m}$ cada una, cada línea contó con cinco trampas de caída y cuatro trampas de cilindro para la captura de saurios y ofidios. Las líneas de trampa fueron establecidas en tramos pequeños $(10 \mathrm{~m})$, con el objeto de abarcar más ambientes e incrementar la posibilidad de capturar más especies (Heyer et al. 1994).

Durante las horas de no revisión de las trampas terrestres se realizaron caminatas sobre senderos en longitudes variables utilizando la técnica descrita por Casas-Andreu et al. (1991), modificada de acuerdo con los hábitos de cada especie (hora de inicio y fin de la actividad). El 


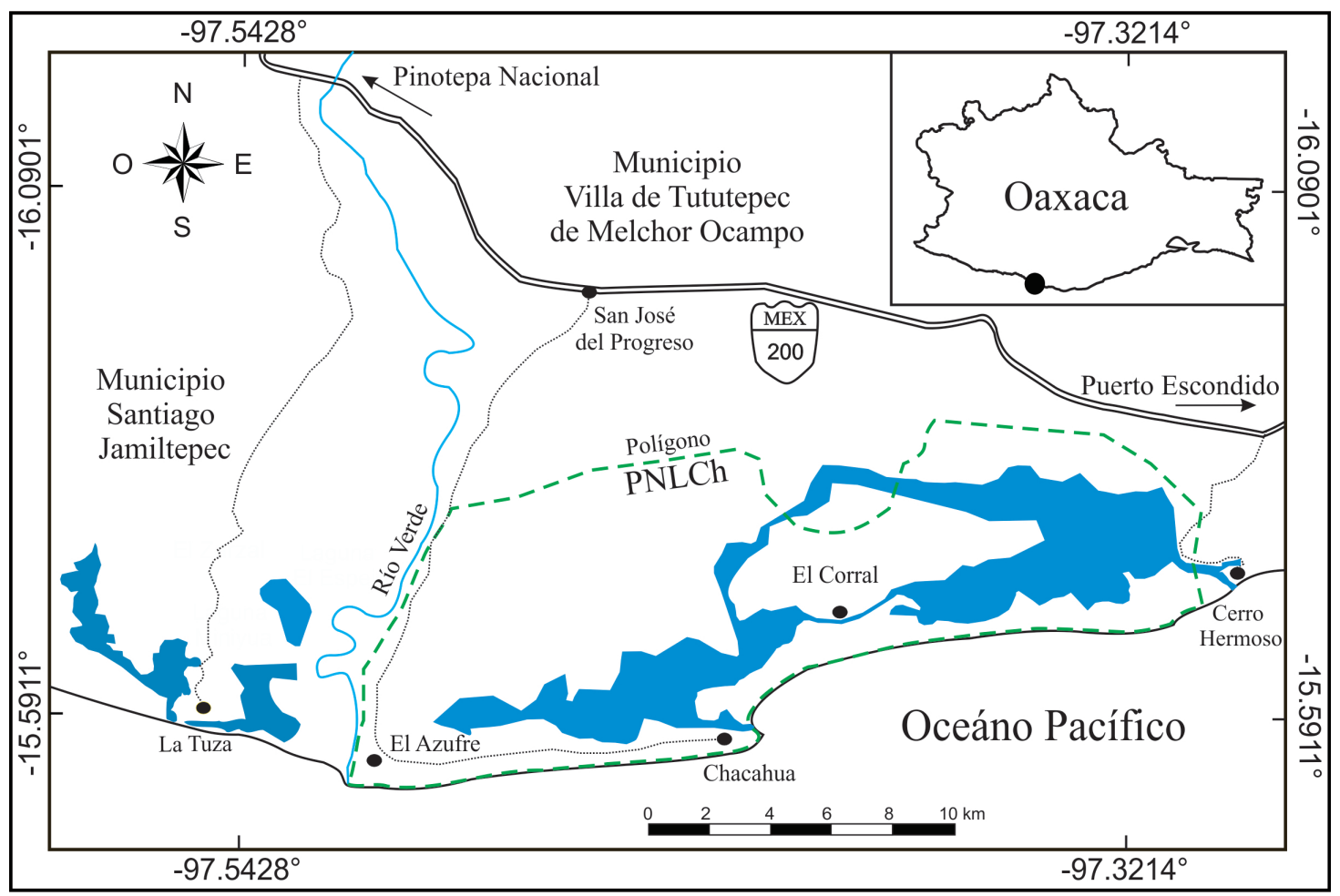

Figura 1. Ubicación del Parque Nacional Lagunas de Chacahua y La Tuza de Monroy, Oaxaca.

horario de muestreo para especies con actividad diurna, que generalmente son los reptiles, fue de 09:00 a 13:00 h y de 15:00 a 18:00 h (Vite-Silva et al. 2010). Algunos organismos con actividad diurna fueron capturados manualmente, mientras que para la captura de las especies venenosas (por ejemplo, géneros Agkistrodon, Crotalus y Porthidium) se usaron ganchos y pinzas herpetológicas. La búsqueda de organismos incluyó diferentes tipos de microhábitats; bajo rocas, sobre y bajo troncos y a orillas de los cuerpo de agua, entre otros (Casas-Andreu et al. 1991). El horario para buscar las especies con actividad nocturna se estableció de las 19:00 a las 23:00 h, y fue dirigido esencialmente para el muestreo de los anfibios $(\mathrm{Ca}-$ sas-Andreu et al. 1991). Para la colecta de estos últimos organismos se utilizó una red de malla con mango debido a la conducta de permanecer en cuerpos de agua, y lámparas de manos libres para facilitar su captura y manejo.

Los individuos colectados en las trampas y observados en los transectos fueron identificados directamente in situ y posteriormente liberados. No se aplicaron técnicas de captura-recaptura para evitar posibles impactos negativos sobre las poblaciones silvestres y por no formar parte de los objetivos de este estudio.
Todos los ejemplares fueron fotografiados y reconocidos a nivel de especie con las diferentes claves dicotómicas para cada grupo (Flores-Villela et al. 1995, Köhler 2008, 2011); y con el apoyo de guías de campo ilustradas (Campbell 1998, Lee 2000, McCranie \& Wilson 2002, Calderón-Mandujano et al. 2005). La nomenclatura taxonómica se basó en Adalsteinsson et al. (2009); Flores-Vi1lela \& Canseco-Márquez (2004); Faivovich et al. (2005, 2010); Frost et al. (2006, 2009), Hynková et al. (2009), y Köhler et al. (2014). Se identificaron las especies prioritarias de anfibios y reptiles, de acuerdo a la legislación nacional (NOM-059-SEMARNAT-2010, Diario Oficial de la Federación 2010) e internacional (la Lista Roja de la Unión Internacional para la Conservación de la Naturaleza (UICN). Se incluyó el cálculo de vulnerabilidad de especies (EVS por sus siglas en inglés) propuesto por Wilson et al. (2013), el cual proporciona un estatus de conservación para todas las especies en términos de su vulnerabilidad, considerando tres categorías principales para los reptiles (distribución geográfica, tipos de vegetación que ocupa y grado de persecución humana) y para los anfibios (distribución geográfica, tipo de vegetación que ocupa y modo de reproducción) estableciendo pun- 
tajes de vulnerabilidad que van de bajo (3-9), medio (1013) a alto (14-20).

Análisis de datos. Se elaboraron curvas de acumulación de especies de manera independiente para los anfibios y reptiles con la información obtenida en campo para determinar la precisión del esfuerzo de muestreo, utilizando el estimador Chao 2 (Colwell 2009). Adicionalmente, se tomaron en cuenta los algoritmos que emplean proporciones de especies que aparecen sólo una vez (singletons), así como las que aparecen dos veces (doubletons), en el supuesto de que cuando estas dos líneas se cruzan, el inventario es considerado completo (Jiménez-Valverde \& Hortal 2003). La curva de acumulación de especies se generó con ayuda del programa EstimateS v7.5.0 (Colwell 2009).

Se utilizaron los datos de riqueza y abundancia por época del año para estimar el índice de diversidad de Shannon-Wiener $\left(H^{\prime}\right)$, los valores obtenidos se compararon con pruebas de $t$ de Hutcheson para detectar diferencias significativas entre la temporada de sequía y lluvias (Hutcheson 1970).

Para comparar los patrones de abundancia de especies entre épocas del año, se elaboraron curvas de Whittaker o de intervalo-abundancia (Feisinger 2003, Magurran 2004).

\section{RESULTADOS}

Se registró un total de 669 individuos que corresponden a 52 especies, 47 géneros y 26 familias de anfibios y reptiles. El orden de los anfibios representó el $23.07 \%$ al registrar 12 especies, mientras que el orden de los reptiles constituyó el $76.9 \%$ del total de especies encontradas al registrar 40 especies (Cuadro 1). Las familias mejor representadas fueron Colubridae con 10 especies, Dipsadidae e Hylidae con cuatro especies respectivamente.

La curva de acumulación de los anfibios de acuerdo al estimador empleado (Chao 2) predice un potencial de 16 especies, asimismo, los algoritmos especies únicas y especies duplicadas nunca se sobreponen, indicando la necesidad de incrementar el número de muestreos para completar el inventario (Figura 2). Respecto a los reptiles, la curva de acumulación mostró un comportamiento similar, con una tendencia al aumento del número de especies registradas pero sin alcanzar la asíntota, indicando

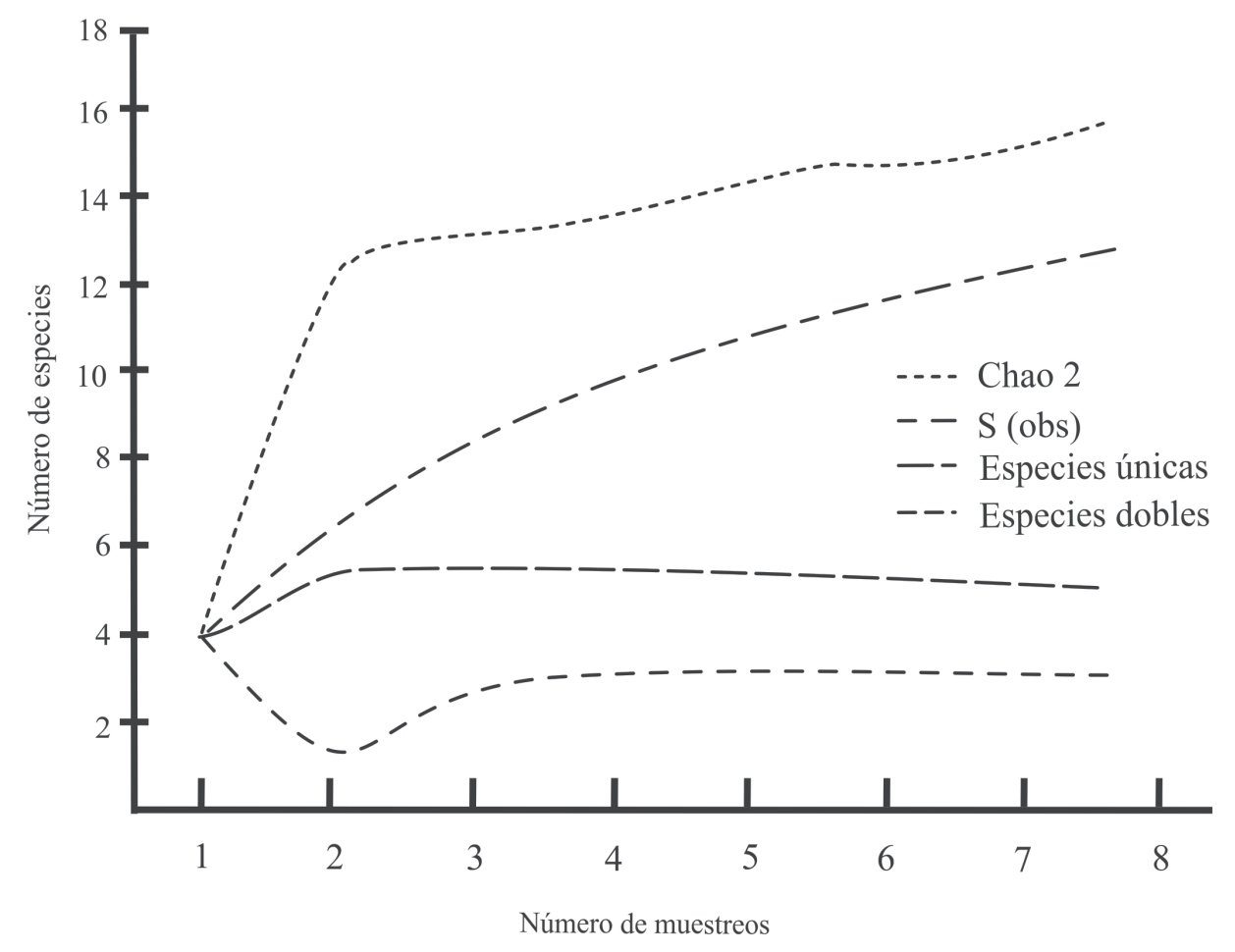

Figura 2. Curva de acumulación de especies de anfibios observadas y esperadas (Chao 2) en el Parque Nacional Lagunas de Chacahua y La Tuza de Monroy, Oaxaca. 
Cuadro 1. Lista de la herpetofauna en las localidades del Parque Nacional Lagunas de Chacahua y La Tuza de Monroy, Oaxaca. $\mathrm{Pr}=$ Protección especial, $\mathrm{SE}=$ Sin Estatus; EVS = Puntaje de vulnerabilidad ambiental de la especie (Siglas en inglés); $\mathrm{B}=$ Bajo, $\mathrm{M}=$ Medio, $\mathrm{A}=$ Alto. La clave (letras) de las especies se relacionan con la figura 4.

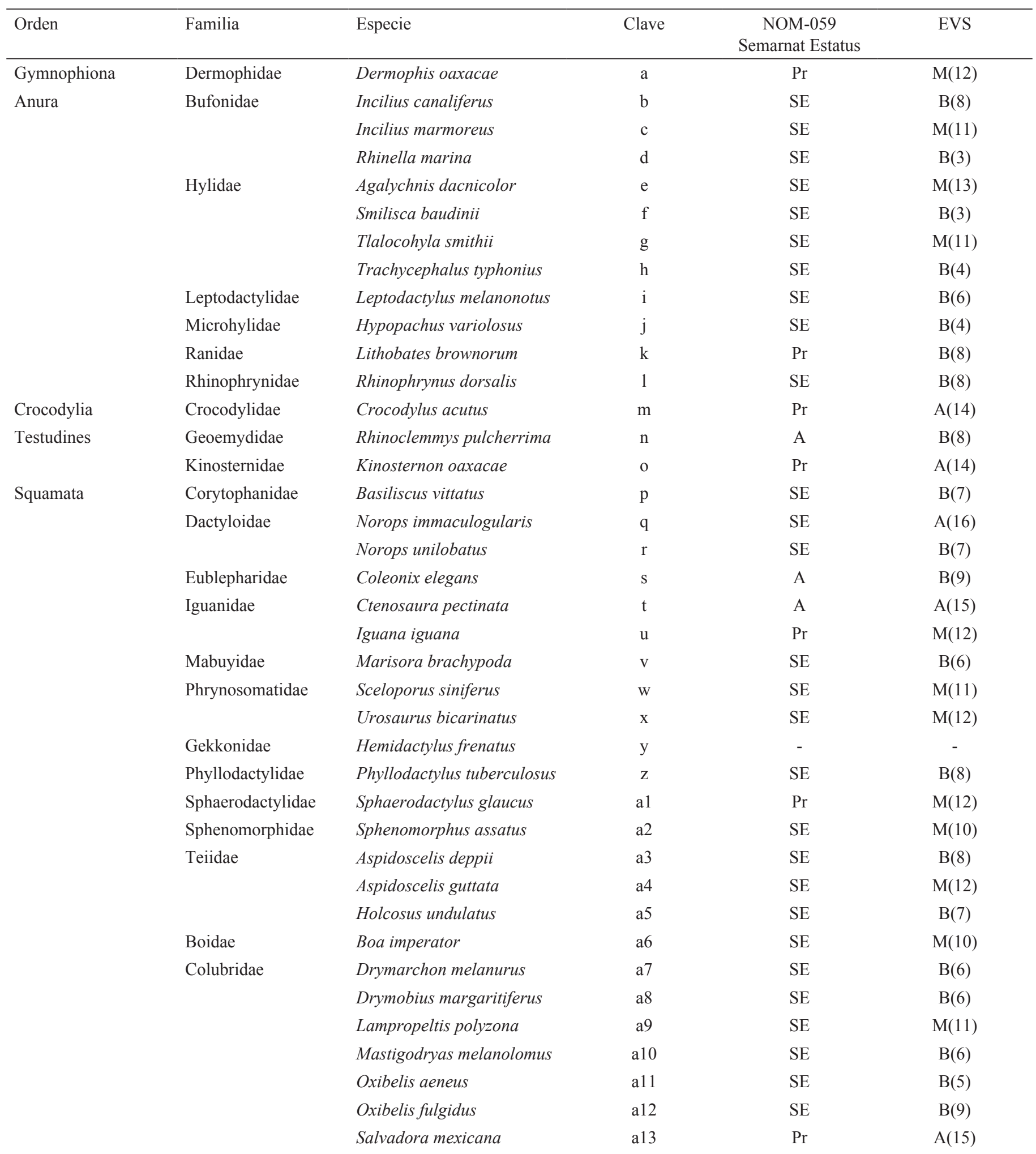


Cuadro 1. Continúa.

\begin{tabular}{|c|c|c|c|c|c|}
\hline Orden & Familia & Especie & Clave & $\begin{array}{c}\text { NOM-059 } \\
\text { Semarnat Estatus }\end{array}$ & EVS \\
\hline & & Stenorrhina freminvillii & a14 & SE & $\mathrm{B}(7)$ \\
\hline & & Trimorphodon biscutatus & a16 & SE & $\mathrm{B}(7)$ \\
\hline & Dipsadidae & Conophis vitattus & a17 & SE & $\mathrm{B}(7)$ \\
\hline & & Leptodeira nigrofasciata & $\mathrm{a} 20$ & SE & $\mathrm{B}(8)$ \\
\hline & & Manolepis putnami & a21 & SE & $\mathrm{M}(13)$ \\
\hline & Leptotyphlopidae & Epictia bakewelli & a22 & SE & $\mathrm{M}(11)$ \\
\hline & Loxocemidae & Loxocemus bicolor & a23 & $\operatorname{Pr}$ & $\mathrm{M}(10)$ \\
\hline
\end{tabular}

la probabilidad de agregar más especies si se intensifica el periodo de muestreo. El comportamiento de los algoritmos especies únicas y especies duplicadas no llegan a sobreponerse debido al registro constante de especies únicas (Figura 3). Durante la temporada de lluvias se registraron 10 especies de anfibios, mientras que en el caso de los reptiles se registraron siete especies exclusivas en cada estación (sequía y lluvias).

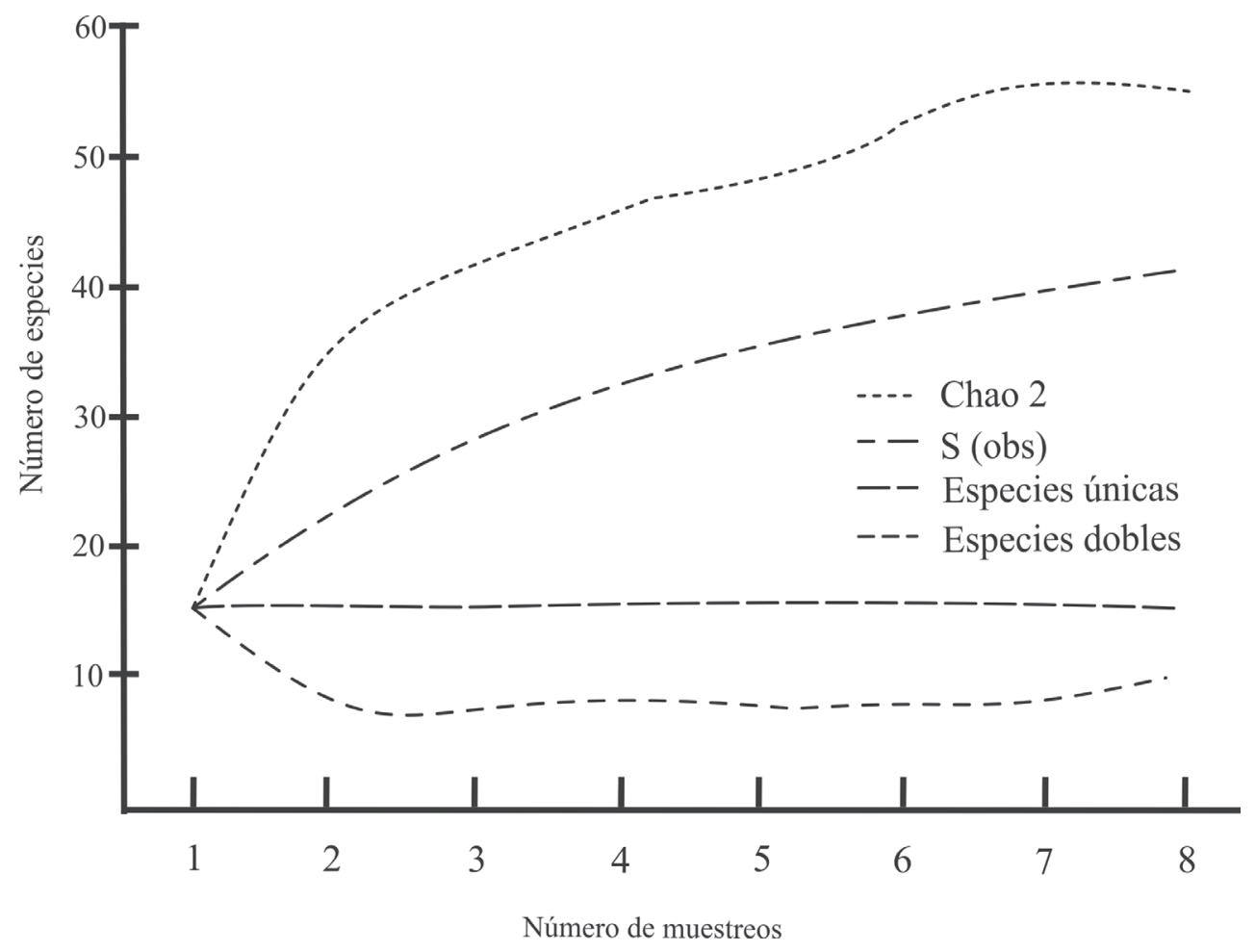

Figura 3. Curva de acumulación de especies de reptiles observadas y esperadas (Chao 2) en el Parque Nacional Lagunas de Chacahua y La Tuza de Monroy, Oaxaca. 
De las 52 especies encontradas, solamente 15 se encuentran en alguna categoría de riesgo según la NOM059-SEMARNAT-2010; 12 especies se encuentran como sujetas a protección especial $(\mathrm{Pr}) \mathrm{y}$ tres especies como amenazadas (Diario Oficial de la Federación 2010). Respecto a la UICN, esta incluye un número mayor (24) de especies en su lista roja; y los apéndices CITES solamente incluyen cuatro species, Iguana iguana, Boa imperator (citada como B. constrictor), Heloderma horridum y Loxocemus bicolor. De acuerdo con el EVS, 26 especies están consideradas como de baja vulnerabilidad, 17 especies como de media vulnerabilidad y ocho especies como de alta vulnerabilidad. Las especies Ctenosaura pectinata, Salvadora mexicana y Crotalus culminatus presentaron en este estudio el mayor grado de vulnerabilidad (puntaje $=15$ ).

El número de especies de anfibios y reptiles registradas fue mayor durante la época de lluvias con 45 especies, mientras que en la época de sequía disminuyó a 34 . Los anfibios registraron mayor diversidad $\left(H^{\prime}=0.98\right)$ en la época de lluvias, mientras que en el caso de los reptiles $\left(H^{\prime}=1.14\right)$ se registró durante la época de sequía. La prueba de Hutcheson corrobora que la diversidad de los anfibios entre épocas fue estadísticamente diferente $(t=$
1.22 , g. $1 .=406, P>0.05)$ no así en el caso de los reptiles, donde no se observaron diferencias significativas $(t=$ 3.07, g. $1 .=489, P<0.05$ ).

El índice de Simpson evidenció una mayor dominancia de los anfibios en la época de lluvias $(t=0.39)$, mientras que en los reptiles la mayor dominancia se observó en la época de sequía $(t=0.15)$.

Las curvas de intervalo-abundancia mostraron un marcada dominancia de especies que se ven favorecidas por las actividades humanas como Rhinella marina y Hemidactylus frenatus, ésta última una especie exótica; mientras que las especies poco frecuentes en las cuatro localidades fueron Dermophis oaxacae, Trachycephalus typhonius, Sphaerodactylus glaucus, Porthidium dunni y Oxybelis fulgidus al registrarse en una sola ocasión y en sitios particulares (Figura 4).

\section{DISCUSIÓN}

Si bien el estado de Oaxaca es considerado como la entidad federativa con la mayor diversidad de anfibios y reptiles (Mata-Silva et al. 2015) en México, los estudios de estos vertebrados se han concentrado mayoritariamente

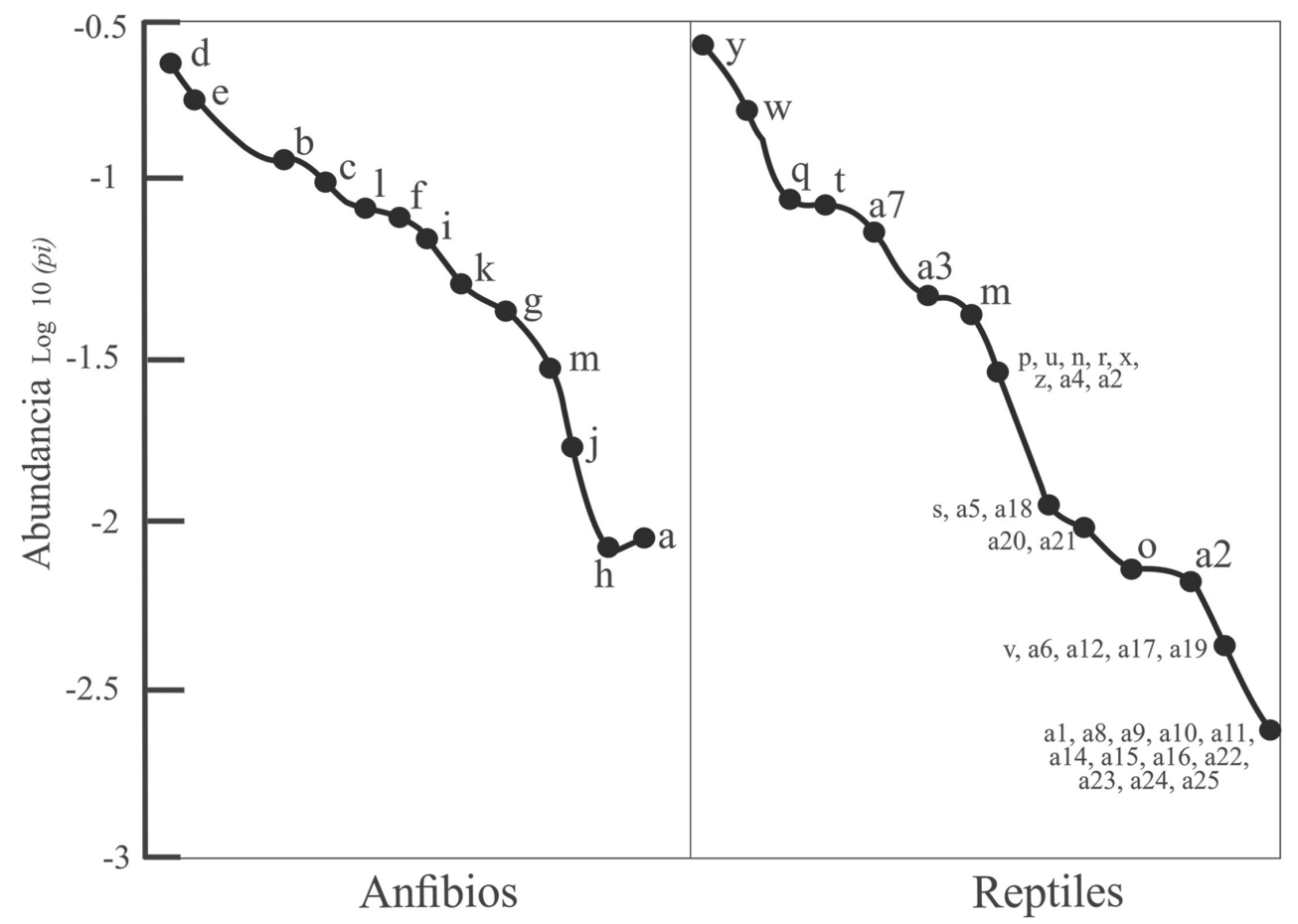

Figura 4. Curva de intervalo-abundancia de la composición de anfibios y reptiles en el Parque Nacional Lagunas de Chacahua y La Tuza de Monroy, Oaxaca en la temporada de lluvias. La clave de las especies (números) están listadas en el Cuadro 1. 
en la parte noreste y este del estado (Casas-Andreu et al. 2004), mientras que regiones como la Sierra Madre del Sur y la planicie costera continúan con escasa información. El presente trabajo aporta información importante sobre la riqueza de anfibios y reptiles de la planicie costera, particularmente del PNLCh, donde el número de especies registradas representa $11.76 \%$ (52 especies) del total de especies de anfibios y reptiles del estado (442 especies; Mata-Silva et al. 2015) y $4.32 \%$ del total de especies en México (1203 especies; Wilson et al. 2013a, b). Respecto al número de especies registradas en la provincia Planicie Costera del Pacífico (93 especies; Mata-Silva et al. 2015), el sitio de estudio presenta el 55.9\% de las especies de anfibios y reptiles de la provincia, resaltando su importancia del sitio en cuanto a la riqueza herpetofaunística regional.

La riqueza herpetofaunística del PNLCh, es similar a la de la región de Nizanda, ubicada al este de la entidad con 59 especies (Barreto-Oble 2000) y mayor que la del municipio de Pluma-Hidalgo, al sur del estado con 44 especies (Caviedes-Solis 2009) y la del Cerro Guiengola y la región norte de la Laguna Inferior en el Istmo de Tehuantepec con 40 y 49 especies respectivamente (MartínRegalado et al. 2011, Rioja-Paradela et al. 2013). Ésta riqueza incluso fue superior comparada con otras áreas similares en características ambientales como Chamela, Jalisco donde se reportan 34 especies de anfibios y reptiles (García \& Cabrera Reyes 2008).

De acuerdo al estimador Chao 2, la curva de acumulación de especies de anfibios alcanzó el $81.2 \%$ de las especies previstas, los registros de los algoritmos único y duplicados no se sobrepusieron indicando que el inventario de estas especies está incompleto. En el caso de los reptiles, la curva de acumulación de especies alcanzó el $83.7 \%$ de las especies previstas por el mismo estimador Chao 2 y el comportamiento de los algoritmos únicos y duplicados no mostraron tendencia a la sobreposición probando que el inventario de reptiles tampoco está completo y que es muy probable que de incrementarse el esfuerzo de muestreo se registrarían otras especies de reptiles.

Cabe mencionar que en este trabajo no se consideraron los registros de las tortugas marinas que se distribuyen en esta zona (Dermochelys coriacea, Lepidochelys olivacea, Chelonia agassizi y Eretmochelys imbricata; Raymundo González 2010) dado que el muestreo no se realizó sobre la franja de playa, por lo que también se excluyó la presencia de Hidrophys platura cuyos registros de varamiento son frecuentes en la costa de Oaxaca (Meraz 2008).
La riqueza observada en reptiles fue mayor que la de anfibios y coincide con la lista generada por Casas-Andreu et al. (2004) para la provincia fisiográfica Costa de Oaxaca; adicionalmente, en este trabajo comprobamos que la composición de especies de la región de Chacahua y La Tuza de Monroy se caracteriza por poseer un número considerable de especies relativamente poco abundantes y una cantidad de especies con abundancia relativa alta (Gardner et al. 2007, García \& Cabrera Reyes 2008, Ricklefs 2001).

Para la mayoría de las especies de anfibios y reptiles, la temporada de lluvias propicia un ambiente adecuado para el inicio de la reproducción y aumento en la abundancia de presas potenciales, lo que influye directamente sobre el aumento de la diversidad herpetofaunística (Caviedes-Solis 2009). Por el contrario, en la temporada de sequía el registro se ve afectado por diversas causas como el cambio de las condiciones climáticas y la disminución de los recursos, por lo tanto, algunas especies reducen sus actividades, especialmente los anfibios. Nuestros resultados muestran un efecto marcado de la estacionalidad sobre la diversidad de anfibios durante la época de lluvias en contraste con lo observado en los reptiles, al observar que las tendencias temporales registradas fueron más evidentes para los anfibios durante la época de lluvias de acuerdo con el índice de Shannon-Wiener. Probablemente esta diferencia se pueda atribuir a la contrastante fisiología y la estrecha relación que existe entre la presencia de los cuerpos de agua y los ciclos biológicos de los anfibios, provocando que la ocurrencia de especies y actividad difiera por influencia de la estacionalidad ambiental (Androne et al. 2001, García \& Cabrera Reyes 2008). El efecto de la estacionalidad en las lluvias sobre las fluctuaciones en las comunidades de anfibios, ya ha sido documentado en otras áreas tropicales, en especial en aquellas regiones marcadamente estacionales (Duellman \& Thomas 1996, García \& Cabrera Reyes 2008).

En Chacahua, si bien existen cuerpos lagunares, éstos presentan cambios dinámicos a lo largo del año en sus características fisicoquímicas (Ahumada-Sempoal \& RuizGarcía 2008), aunado a la contrastante fisionomía de la selva baja caducifolia que presenta un marcado periodo de sequía con una duración de 5 a 7 meses y la presencia de especies arbóreas que pierden las hojas durante este periodo, factores que generan el incremento en la temperatura del suelo, lo que constituye una limitante en las condiciones óptimas de hábitat requerido por los anfibios ( Lott et al. 1987). 
Aunque se sabe que la estructura de la vegetación no define los atributos de la comunidad de anfibios y reptiles por completo y considerando que algunos de los organismos que componen a esta comunidad tienen hábitos fosoriales, terrestres, acuáticos y arborícolas (García \& Ceballos 1994), es posible que las especies de anfibios registradas en este estudio respondan en mayor grado a los cambios en la estructura de la vegetación que los reptiles, coincidiendo con lo reportado en sitios como Chamela, Jalisco donde la vegetación predominante también está constituida por selva baja caducifolia (García \& Cabrera Reyes 2008).

Por otro lado, existen reportes sobre las ventajas que ofrecen los factores climáticos como temperatura, precipitación, fotoperiodo y la disponibilidad de alimento a las especies nativas e invasoras de fácil adaptación a distintas condiciones y con dietas insectívoras generalistas (Romero-Mayen 2008), sobre todo cuando se ven favorecidas por la perturbación del hábitat como consecuencia de las actividades humanas. Lo anterior se ve reflejado en el presente estudio, ya que las especies de anfibios y reptiles más dominantes fueron las especies Rhinella marina y Hemidactylus frenatus. Respecto a $H$. frenatus, no se sabe con certeza cuál ha sido el impacto que ha generado sobre la fauna local; sin embargo, es probable que afecte a otras especies nativas al competir por alimento y espacio. Existe también la posibilidad de que $H$. frenatus forme parte de la cadena trófica de algunos vertebrados en estos ecosistemas, como ha sido reportado en otras partes de la república mexicana (Rojas-González y Wakida-Kusunoki 2012) u otras entidades en Mesoamérica (Mata-Silva et al. 2013). Un situación muy similar fue observada por Vite-Silva et al. (2010) en varios ambientes de la Reserva de la Biósfera Barranca de Meztitlán, al reconocer los patrones de abundancia de anfibios y reptiles de esa región.

En relación a la protección de la herpetofauna en el PNLCh, el sistema EVS muestra un puntaje de las especies registradas en este estudio con un decremento de la categoría baja (26 especies) hacia la intermedia (17 especies) y la alta (8 especies), por lo que los esfuerzos de conservación deberán centrarse en aquellas especies que muestran una alta vulnerabilidad. Contrariamente, la legislación nacional sólo categoriza a 15 especies bajo algún estatus de protección, lo que coincide con algunas especies categorizadas por el sistema EVS; sin embargo, omite a otras cuya sobrevivencia podría ser afectada por no considerarse de mediana o alta vulnerabilidad. El caso de Crocodylus acutus como especie altamente vulnerable
(Puntaje EVS= 15) debe ser considerado por la adminstración del Parque Nacional como una prioridad para su conservación. Aunado a esto, el conocimiento y percepción cultural que las comunidades cercanas o aledañas a dicha ANP tienen de los cocodrilos podrían ser un factor fundamental en las estrategias de conservación de esta especie y de la fauna silvestre en general (García-Grajales y Buenrostro-Silva 2015).

Este trabajo agrega la presencia de 5 especies de anfibios y 19 especies de reptiles al listado realizado por Casas-Andreu et al. (2004) en la planicie costera de Oaxaca; Oxybelis fulgidus, Agkistrodon bilineatus (García-Grajales \& Buenrostro-Silva 2011) y Sphaerodactylus glaucus (García-Grajales et al. 2014) como especies distribuidas en la planicie costera central del estado. Symphimus leucostomus podría también estar distribuida en regiones cercanas al PNLCh (García-Grajales et al. 2012), lo que demuestra la probabilidad de incrementar el conocimiento y por ende la necesidad de realizar más estudios acerca de la diversidad, distribución y riqueza biológica de los anfibios y reptiles que habitan en los ambientes tropicales tan escasamente estudiados en el estado de Oaxaca.

El presente estudio contribuye al conocimiento sobre la distribución geográfica de la herpetofauna en México, en particular de la planicie costera central del estado de Oaxaca; asimismo se sugiere la continuidad de estudios en temas relacionados a la estructura, dinámica de poblaciones y de ecología de comunidades que permitan entender claramente la importancia que tienen estos grupos biológicos en la zona y que promuevan suscitar estrategias de conservación más específicas (Lips 1998, ViteSilva et al. 2010). Además, este trabajo pone en evidencia la importancia ecológica que presenta esta región, la cual aún esconde una gran riqueza biológica en cuanto a anfibios y reptiles se refiere.

AGRADECIMIENTOS. Agradecemos a la Universidad del Mar (UMAR) y a la Comisión Federal de Electricidad (CFE) a través del convenio de colaboración (CUP: 2IR0807) por las facilidades y financiamiento de esta investigación. Miguel Antonio-Gutiérrez, Batlin Pineda, Daniela Sigüenza y Manuel Tenorio Salgado apoyaron en el trabajo de campo. Asimismo, agradecemos la hospitalidad y facilidades otorgadas por los habitantes de las comunidades de La Tuza, El Azufre, El Corral y Cerro Hermoso. A la Secretaría de Medio Ambiente y Recursos Naturales por el permiso de colecta científica (SGPA/ DGVS/07772/08) otorgado al primer autor. Dor revisores anónimos contribuyeron a mejorar el contenido del presente trabajo y les agradecemos sus aportaciones. 


\section{LITERATURA CITADA}

Adalsteinsson, S. A., Branch, W. R., Trape, S., Vitt, L. J. \& BlairHedges, S. 2009. Molecular phylogeny, classification, and biogeography of snakes of the family Leptotyphlopidae (Reptilia, Squamata). Zootaxa, 2244: 1-50.

Ahumada-Sempoal, M. A. \& Ruiz García, N. 2008. Características físico-químicas de la laguna Pastoría, Oaxaca, México. Ciencia y Mar, XII(36): 3-17.

Barreto-Oble, D. 2000. Análisis ecológico y distribucional de los anfibios y reptiles de la región de Nizanda, Istmo de Tehuantepec, Oaxaca. Tesis, Fac. de Ciencias, UNAM, México.

Calderón-Mandujano, R., Bahena Basave, H. \& Calmé, S. 2005. Anfibios y reptiles de la Reserva de la Biósfera de Sian Ka'an y zonas aledañas. COMPACT, ECOSUR, CONABIO. Chetumal, Quintana Roo, México.

Campbell, J. A. 1998. Amphibians and reptiles of northern Guatemala, the Yucatan and Belize. University of Oklahoma Press, Oklahoma, USA.

Casas-Andreu, G., Valenzuela-López, G. \& Ramírez-Bautista, A. 1991. Cómo hacer una colección de Anfibios y Reptiles. Instituto de Biología. UNAM. Cuadernos No. 10, México, D.F.

Casas-Andreu, G., Méndez de la Cruz, F. R. \& Camarillo, J. L. 1996. Anfibios y reptiles de Oaxaca. Lista, distribución y conservación. Acta Zoológica Mexicana (nueva serie), 69: 1-35.

Casas-Andreu, G., Méndez de la Cruz, F. R. \& Aguilar-Miguel, X. 2004. Anfibios y reptiles. Pp. 375-390. In A. J. García-Mendoza, M. J. Ordoñez \& M. Briones-Salas (Eds.). Biodiversidad de Oaxaca, Instituto de Biología, UNAM-Fondo Oaxaqueño para la Conservación de la Naturaleza-World Wildlife Conservation Fund, México.

Caviedes-Solis, L. W. 2009. Estudio herpetofaunístico del municipio de Pluma Hidalgo, Oaxaca, México. Tesis, Fac. de Ciencias, Universidad Nacional Autónoma de México, México.

Collwell, R. K. 2009. EstimateS: Statistical estimation of species richness and shared species from samples. Version 8.2. Users guide and application. Available at: http://purl.oclc.or/estimates (accesado en Julio de 2013).

Diario Oficial de la Federación. 2010. Norma Oficial Mexicana NOM-050-SEMARNAT-2010, que determina las especies de flora y fauna silvestres, terrestres y acuáticas, endémicas, amenazadas, en peligro de extinción y sujetas a protección especial. Órgano del Gobierno Constitucional de los Estados, Gobierno Federal, México.

Duellman, W. E. \& Thomas, R. 1996. Anuran amphibians from seasonally dry forest in Southeastern Peru and comparisons of the anuran among sites in the upper Amazon Basin. Occasional Papers of Museum Natural History, University of Kansas, 180: 1-34.

Faivovich, J., Hadad, C. F. B., García, P. C. A., Frost, D. R., Campbell, J. A. \& Wheeler, W. C. 2005. Systematics review of the frog family Hylidae, with especial reference to Hylinae: Phylogenetic analysis and taxonomic revision. Bulletin of the American Museum of Natural History, 294: 1-240.

Faivovich, J., Haddad, C. F. B., Baeta, D., Jungfer, K. H., Álvares, G. F. R., Brandao, R. A., Sheil, C., Barrientos, L. S., BarrioAmorós, C. L., Cruz, C. A. G. \& Wheeler, W. C. 2010. The phylogenetic relationships of the charismatic poster frogs, Phyllomedusinae (Anura, Hylidae). Cladistics, 26: 227-261.

Feisinger, P. 2003. El diseño de estudio de campo para la conservación de la biodiversidad. FAN, Santa Cruz de la Sierra, Bolivia. $242 \mathrm{p}$.

Flores-Villela, O. A. \& Canseco-Márquez L. 2004. Nuevas especies y cambios taxonómicos para la herpetofauna de México. Acta Zoológica Mexicana (nueva serie), 20 (2): 115-144.

Flores-Villela, O. A., Mendoza-Quijano, F. \& González Porter, G. 1995. Recopilación de claves para la determinación de anfibios y reptiles de México. Publicaciones Especiales del Museo de Zoología, UNAM, 10: 1-285.

Flores-Villela, O., Ochoa-Ochoa, L. M. \& Moreno, C. E. 2005. Variaciones latitudinales y longitudinal de la riqueza de especies y la diversidad beta de la herpetofauna mexicana. Pp. 143-151. In: G., Halffter, J. Soberón, P. Koleff \& A. Melic (Eds.). Sobre diversidad biológica: el significado de las diversidades alfa, beta y gamma. Monografías 3er. Milenio, 4. Sociedad Entomológica Aragonesa/ Comisión para el Conocimiento y Uso de la Biodiversidad / Diversitas/ Consejo Nacional de Ciencia y Tecnología, México, D.F.

Frost, D. R., Grant, T., Faivovich, J., Bain, R. H., Haas, A., Haddad, C. F. B., De Sá, R. O., Channing, A., Wilkinson, M., Donnellan, S. C., Raxworthy, C. J., Campbell, J. A., Blotto, B. L., Moler, P., Drewes, R. C., Nussbaum, R. A., Lynch, J. D., Green, D. M. \& Wheeler, W. C. 2006. The amphibian tree of life. Bulletin of the American Museum of Natural History, 297: 1-370.

Frost, D. R., Mendelson, J. R. \& Pramuk, J. 2009. Further notes on the nomenclature of Middle American Toads (Bufonidae). Copeia, 2009: 418.

García, E. 1988. Modificaciones al sistema de clasificación climática de Köppen (Para adaptarlo a las condiciones de la República mexicana). 4 ed., Offset Larios, México.

García, A. \& Ceballos, G. 1994. Guía de campo de los reptiles y anfibios de la costa de Jalisco, México. Fundación Ecológica Cuixmala-Instituto de Biología, UNAM, México.

García, A. \& Cabrera Reyes, A. 2008. Estacionalidad y estructura de la vegetación en la comunidad de anfibios y reptiles de Chamela, Jalisco, México. Acta Zoológica Mexicana (nueva serie), 24: 91-115.

García-Grajales, J. 2008. Herpetología-Notas para el estudio de los anfibios y reptiles en Oaxaca. Ciencia y Mar, XII (34): 47-56.

García-Grajales, J. \& Buenrostro-Silva, A. 2011. Ampliación de la distribución geográfica de Oxybelis fulgidus (Serpentes: Colubridae) y Agkistrodon bilineatus (Serpentes: Viperidae) en la planicie costera central de Oaxaca, México. Acta Zoológica Mexicana (n. s.), 27: 491-495.

García-Grajales, J. \& Buenrostro-Silva, A. 2015. Apreciación local acerca del cocodrilo americano (Crocodylus acutus) en comunidades rurales aledañas al Parque Nacional Lagunas de Chacahua (Oaxaca, México). Revista de Etnobiología, 13: 73-80.

García-Grajales, J., Buenrostro-Silva, A. \& Mata-Silva, V. 2014. Range extension of the least gecko, Sphaerodactylus glaucus Cope, 1865 (Squamata: Sphaerodactylidae) in Oaxaca, Mexico. Check List, 10: 205-206.

García-Grajales, J., López-López, Y., Buenrostro-Silva, A. \& Mata-Silva, V. 2012. Symphimus leucostomus Cope, 1869 (Reptilia: Squamata: Colubridae): Distribution extension in the Pacific lowlands of Oaxaca, Mexico. Check List, 8: 917-918. 
Gardner, T. A., Fitzherbert, E. B., Drewes, R. C., Howell, C. \& Caro, T. 2007. Spatial and temporal patterns of abundance and diversity of an east African leaf litter amphibian fauna. Biotropica, 39: 105-113.

Halffter, G. 2005. Towards a culture of biodiversity conservation. Acta Zoológica Mexicana (nueva serie), 21(2): 133-153.

Halffter, G. 2007. Reservas archipiélago: Un nuevo tipo de área protegida. Pp. 281-286. In: G. Halffter, S. Guevara y A. Melic (Eds.). Hacia una cultura de conservación biológica. SEA, CONABIO,CONANP, CONACYT, INECOL, UNESCO-Mab \& Ministerio de Medio Ambiente-Gobierno de España. m3m. Monografías del Tercer Milenio, vol. 6. S.E.A., Zaragoza.

Hernández-Santos, I. 2009. Propuesta de programa para el Manejo Integral de la Zona Costera. Caso: Municipio de Villa de Tututepec de Melchor Ocampo, Oaxaca, México. Tesis, Universidad del Mar, Puerto Ángel, Oaxaca.

Heyer, W. R., Donelly, M. A., McDiarmid, R. W., Hayek, L. A. C. \& Foster, M. S. 1994. Measuring and monitoring biological diversity. Standard methods for amphibians. Smithsonian Institution Press, Washington and London.

Hynková, I., Starostová, Z. \& Frinta, D. 2009. Mitochondrial DNA variation reveals recent evolutionary history of main Boa constrictor clades. Zoological Science, 26: 623-631.

Hutcheson, K. 1970. A test for comparing diversities based on the Shannon formula. Journal of Theorical Biology, 29: 151-154.

Köhler, G. 2008. Reptiles of Central America. $2^{\text {nd }}$ edition. Herpeton Verlag, Offenbach, Germany.

Köhler, G. 2011. Amphibians of Central America. Herpeton Verlag, Offenbach, Germany.

Köhler, G., Gómez Trejo Pérez, R., Petersen, C. B. P. \& Méndez de la Cruz, F. R. 2014. A revision of the Mexican Anolis (Reptilia, Squamata, Dactyloidae) from the Pacific versant west of the Isthmus de Tehuantepec in the states of Oaxaca, Guerrero, and Puebla, with descriptions of six new species. Zootaxa, 3862: 001210.

Lira, T. I. \& Naranjo, E. 2005. Ampliación de la distribución geográfica de Tapirus bairdii Gill 1865 (Perisodactila: Tapiridae) en México. Acta Zoológica Mexicana (n. s.), 21: 107-110.

Lott, E. J., Bullock, S. H. \& Solis-Magallanes, A. 1987. Floristic diversity and structure of upland and arroyo forest of coastal Jalisco. Biotropica, 19: 228-235.

Jiménez-Valverde, A. \& Hortal, J. 2003. Las curvas de acumulación de especies y la necesidad de evaluar la calidad de los inventarios biológicos. Revista Ibérica de Aracnología, 8: 151-161.

Lee, J. C. 2000. A field guide to the Amphibians and Reptiles of the Maya World. Cornell University Press.

Lips, K. R. 1998. Decline of a tropical montane amphibian fauna. Conservation Biology, 12: 106-117.

Magurran, A. E. 2004. Measuring biological diversity. Blackwell, Oxford. $256 \mathrm{p}$.

Martín-Regalado, C. N., González-Ugalde, R. M. \& Cisneros Palacios, E. 2011.Herpetofauna del cerro Guiengola, Istmo de Tehuantepec, Oaxaca. Acta Zoológica Mexicana (n. s.), 27: 359-376.

Mata-Silva, V., Wilson, L. D. \& Johnson, J. D. 2013. Hemidactylus frenatus. Predation. Herpetological Review, 44: 508-509.

Mata-Silva, V., Johnson, J. D., Wilson, L. D. \& García-Padilla, E. 2015. The herpetofauna of Oaxaca, Mexico: composition, phy- siographic distribution, and conservation status. Mesoamerican Herpetology, 2: 6-62.

McCranie, J. R. \& Wilson, L. D. 2002. The amphibians of Honduras. Society for the Study of amphibians and reptiles. Contributions to Herpetology, 19: 1-625.

Mena-Correa, A. L. 2008. Estudio de la comunidad de anfibios y reptiles en dos remanentes medianamente alterados, adyacentes al macizo de selvas en la Reserva de la Biosfera "Los Tuxtlas", Veracruz. Tesis de Licenciatura, Fac. de Ciencias, Universidad Nacional Autónoma de México. México, D. F.

Meraz, H. J. 2008. Pelamis platurus (Reptilia: Elapidae) en la costa de Oaxaca. Ciencia y Mar, XI (33): 39-43.

Navarro, S. A. G., García-Trejo, E. A., Townsend Peterson, A. \& Rodríguez-Contreras, V. 2004. Aves. Pp. 391-421. In: A. J. García-Mendoza, M. J. Ordoñez y M. Briones-Salas (Eds.). Biodiversidad de Oaxaca, Instituto de Biología, UNAM-Fondo Oaxaqueño para la Conservación de la Naturaleza-World Wildlife Conservation Fund, México.

Pérez-Delgado, P. E. 2002. Estado de conservación de la vegetación del Parque Nacional Lagunas de Chacahua: propuesta para su rehabilitación. Pp. 29-37. In: M. Alfaro y G. Sánchez (eds.). Chacahua: Reflejos de un Parque, Comisión Nacional de Áreas Naturales Protegidas, Programa de las Naciones Unidas, Secretaría de Medio Ambiente y Recursos Naturales, Plaza y Valdez, México.

Raymundo-González, I. 2010. Caracterización de las tortugas prietas (Chelonia mydas) del complejo lagunar Chacahua-Pastoría. Tesis, Universidad del Mar, Puerto Ángel, Oaxaca.

Ricklefs, R. E. 2001. The economy of nature. Fifth edition. Freeman Bookshelf. Zsaro, R. C. y S. C. Belfit. 1986. Herpetofaunal use of a desert riparian islands and its adjacent scrub habitat. Journal of Wildlife Management, 50: 752-761.

Rioja-Paradela, T., Carrillo Reyes, A., Castañeda, G. \& López, S. 2013. Diversidad herpetofaunística al norte de la laguna Inferior, Istmo de Tehuantepec, Oaxaca, México. Acta Zoológica Mexicana (n. s.), 29: 574-595.

Rojas-González, R. I., \& Wakida-Kusunoki, A. T. 2012. Hemidactylus frenatus. Predation. Herpetological Review, 43: 133.

Rzedowki, J. 1978. Vegetación de México. Limusa, México. 432 p.

Romero-Mayén, A. R. 2008. Impacto ecológico de la herpetofauna introducida e invasora en la República Mexicana. Tesis de Licenciatura, Facultad de Ciencias, Universidad Nacional Autónoma de México.

Torres-Colín, R. 2004. Tipos de vegetación. Pp. 105-117. In: A. J. García-Mendoza, M. J. Ordoñez y M. A. Briones-Salas (Eds.). Biodiversidad de Oaxaca, UNAM-Fondo Oaxaqueño para la Conservación de la Naturaleza-World Wildlife Fund, México.

Trejo, I. 2004. Clima. Pp. 67-85. In: A. J. García-Menoza, M. J. Ordoñez y M. A. Briones-Salas (Eds.). Biodiversidad de Oaxaca, UNAM-Fondo Oaxaqueño para la Conservación de la NaturalezaWorld Wildlife Fund, México.

Vite-Silva, V. D., Ramírez-Bautista, A. \& Hernández-Salinas, U. 2010. Diversidad de anfibios y reptiles de la Reserva de la Biósfera Barranca de Metztitlán, Hidalgo, México. Revista Mexicana de Biodiversidad, 81: 473-485.

Wilson, L. D., Mata-Silva, V. \& Johnson, J. D. 2013. A conservation reassessment of the reptiles of Mexico based on the EVS measure. Amphibian and Reptile Conservation, 7: 1-47. 